DOI: https://doi.org/10.46296/ig.v4i8edespdic.0052

\title{
CARACTERIZACIÓN DEL ESTADO DE APLICACIÓN DE LAS TÉCNICAS DE MANTENIMIENTO PREDICTIVO DE LA FLOTA ATUNERA INDUSTRIAL QUE OPERA EN EL PACÍFICO ORIENTAL
}

\section{CHARACTERIZATION OF THE STATE OF APPLICATION OF PREDICTIVE MAINTENANCE TECHNIQUES IN THE INDUSTRIAL TUNA FLEET OPERATING IN THE EASTERN PACIFIC}

\author{
Zambrano-Ascencio José Adrián ${ }^{1}$; Pérez-Guerrero Julio Nolberto ${ }^{2}$ \\ ${ }^{1}$ Estudiante de la Maestría en Mantenimiento Industrial, Mención en Gestión Eficiente de \\ Mantenimiento, Instituto de Posgrado, Universidad Técnica de Manabí. Portoviejo, Ecuador. \\ Correo: jzambrano1082@utm.edu.ec. ORCID ID: https://orcid.org/0000-0001-9594-2057. \\ 2 Docente de la Maestría en Mantenimiento Industrial - Departamento de Mecánica, \\ Universidad Técnica de Manabí. Portoviejo, Ecuador. Correo: julio.perez@utm.edu.ec. \\ ORCID ID: https://orcid.org/0000-0003-2769-3209.
}

\begin{abstract}
Resumen
La actividad pesquera es considerada de gran importancia desde un enfoque alimenticio y económico debido a que se desarrolla de manera proporcional al crecimiento poblacional; se considera que el primer eslabón de esta cadena de suministro son las embarcaciones atuneras, siendo esencial para su funcionamiento la gestión de mantenimiento, sobre todo de tipo predictivo, sin embargo, hoy en día no se conoce el estado de los elementos fundamentales de mantenimiento de esta vital flota. Considerando que gran parte de la flota superan los 40 años de edad y que al momento no existen estudios definidos sobre el desarrollo de este proceso de mantenimiento, el objetivo principal es caracterizar el estado de aplicación de las técnicas de mantenimiento predictivo de la flota atunera activa del Pacífico Oriental. La metodología aplicada para esta investigación se fundamentó en la caracterización general de la flota activa del Pacífico Oriental a través de información documental que permitió clasificar a las embarcaciones por banderas y años de antigüedad y del estudio de las variables de gestión de mantenimiento utilizada por las embarcaciones, para lo cual se utilizó una muestra de estudio conformada por 41 embarcaciones que realizan operaciones logísticas en puertos ecuatorianos ubicados en los cantones de Manta y Jaramijó. En general la investigación detalla que la flota resulta antigua incluyendo sus motores diésel y resto de equipos a bordo, la misma que no cuenta con tecnologías actuales de control de indicadores, los mismos que son registrados manualmente en bitácoras físicas.
\end{abstract}

Palabras clave: mantenimiento predictivo, motores diésel, parámetros, control, sistema de lubricación, sistema de enfriamiento, sistema de combustible.

\footnotetext{
Abstract

The fishing activity is considered of great importance from a nutritional and economic perspective because it develops in a proportional way to the population growth; It is considered that the first link in this supply chain are tuna vessels, maintenance management being essential for their operation, especially of a predictive type, however, today the state of the fundamental elements of maintenance is not known. this vital fleet. Considering that a large part of the fleet is over $\mathbf{4 0}$ years old and that at the moment there are no defined studies on the development of this maintenance process, the main objective is to characterize the state of application of predictive

Información del manuscrito:

Fecha de recepción: 04 de octubre de 2021.

Fecha de aceptación: 09 de noviembre de 2021.

Fecha de publicación: 08 de diciembre de 2021.
} 
maintenance techniques in the active tuna fleet. of the Eastern Pacific. The methodology applied for this research was based on the general characterization of the active fleet of the Eastern Pacific through documentary information that allowed to classify the vessels by flags and years of age and the study of the maintenance management variables used by the vessels, for which a study sample made up of 41 vessels that carry out logistics operations in Ecuadorian ports located in the cantons of Manta and Jaramijó was used. In general, the investigation details that the fleet is old, including its diesel engines and other equipment on board, which does not have current indicator control technologies, which are manually recorded in physical binnacle.

Keywords: predictive maintenance, diesel engines, parameters, control, lubrication system, cooling system, fuel system.

\section{Introducción}

Desde hace varias décadas, la actividad pesquera mundial se considera de vital importancia desde el punto de vista alimenticio y económico estimando que entre el año 1961 y 2016 el aumento anual de consumo mundial de pescado comestible presentó una media anual equivalente al $3,2 \%$ superando el crecimiento poblacional del 1,6\%. En el mismo reporte de la FAO se estima que en el año 2016 el número de embarcaciones de pesca alcanzaría unos 4,6 millones, una cifra que se mantendría sin cambios desde el año 2014 (FAO, 2018).

La flota atunera que opera en el Pacifico Oriental está conformada por 10 países, siendo Ecuador el país con mayor la flota atunera que opera en ella debido al número de embarcaciones y capacidad de captura, seguido de México, Estados Unidos, Panamá, Venezuela, Perú, Colombia, Nicaragua, España y El Salvador. Según el Ministerio de Relaciones Exteriores en un reporte sobre el sector atunero ecuatoriano realizado en agosto del 2017 concluyó que la pesca industrial de atún es sumamente importante debido a que genera el $8,71 \%$ de las exportaciones no petroleras, es decir, alrededor de USD 1092 millones en ingresos al Ecuador provenientes del exterior. Siendo Manta, Posorja y Guayaquil sus principales puertos de operaciones, se estima que a nivel nacional se establecen aproximadamente unos 24000 puestos de trabajo directo y alrededor de $120 \quad 000$ empleos indirectos, (EXTERIOR, 2017). Actualmente, la flota del sector atunero ecuatoriano cuenta con 107 
embarcaciones de tipo industrial que aseguran el $60 \%$ del aprovisionamiento de materia prima requerida por la industria nacional. Según datos del INP (Instituto Nacional de Pesca) y de la Comisión Interamericana del Atún Tropical (CIAT) tanto en aguas nacionales como internacionales la flota atunera ecuatoriana capturó en el año 2018 alrededor de 287043 ton de atún (Pacheco, 2019).

Para garantizar la capacidad de operación de esta flota es de vital importancia el mantenimiento de la misma. El mantenimiento va desde el mantenimiento correctivo hasta el mantenimiento basado en la condición pasando por el preventivo. Las últimas publicaciones y estudios privilegian los métodos y técnicas de mantenimiento predictivo sobre el resto de técnicas existentes el cual consiste (García, 2016) en la utilización de herramientas tecnológicas con el objetivo de identificar y monitorear fallas, las mismas que se fundamentan en mediciones de señales físicas como son vibraciones, temperaturas, presiones, fluidos, entre otros. Según (Michala y Lazakis, 2018).
Las señales físicas que establecen la condición de una maquina pueden ser monitoreadas de manera en línea y fuera de línea, con los equipos en marcha y apagados, es decir, que la tecnología actual nos permite llevar registros en tiempo real y al encontrar anomalías en los parámetros monitoreados podemos tomar acciones de manera anticipada a posibles riesgos de fallos mayores. Se requiere fijar unos límites 0 patrones aceptables y comparar dichos límites con los que se presentan las variables analizadas. (Jones y Yu-Hua, 2000).

Entre los métodos más efectivos del mantenimiento predictivo se encuentra el monitoreo de señales físicas propias de los motores diésel instalados a bordo de embarcaciones atuneras como son temperaturas, presiones, vibraciones, entre otros y según estudios actualizados se considera al mantenimiento basado en condición como la mejor técnica de mantenimiento predictivo para gestión de monitoreo de datos y de acuerdo a los señalado por Hernández (2021) los datos recopilados se pueden utilizar para establecer tendencias, predecir 
fallas y calcular la vida útil restante de un activo; la planificación de mantenimiento a un equipo, en especial del motor.

Moreno (2016) manifiesta en uno de sus artículos que el mantenimiento basado en la condición es una metodología que tiene como principal objetivo garantizar el correcto funcionamiento de las máquinas mediante una vigilancia periódica de parámetros relevantes e indicadores de la condición actual y que se ejecuta sin necesidad de desmontajes y revisiones periódicas. Además, los investigadores Martínez y Vanegas (2017) definen al mantenimiento basado en la condición como una técnica que previene el deterioro prematuro 0 acumulativo de un equipo o sus componentes revelado en la variación de los parámetros de funcionamiento, rendimiento, estado, entre otros.

La flota atunera que opera en el Pacífico Oriental está conformada por 280 embarcaciones activas de red de cerco y según datos actualizados de la Comisión Interamericana del Atún Tropical se trata de una flota antigua, cuya edad media equivale a 38 años, por lo tanto, demanda de un mantenimiento efectivo empleando tecnologías actualizadas indistintamente de la antigüedad de los equipos presentes a bordo, sin embargo, no existen estudios 0 certezas de cómo se desarrolla este proceso, lo cual muestra la necesidad de investigar en esta dirección, en tal virtud, el objetivo del trabajo es realizar la caracterización del estado de aplicación de la técnicas de mantenimiento predictivo de la flota atunera industrial que opera en el Pacífico Oriental, lo cual resulta de vital importancia para conocer con exactitud la realidad actual en este campo de estudio, en base al análisis documental.

\section{Materiales y métodos}

La presente investigación se desarrolló siguiendo una metodología que integra varios métodos y técnicas conformada por tres etapas de investigación: a) caracterización general de la flota que opera en el Pacífico Oriental, b) Análisis de la información documental sobre el mantenimiento. La caracterización general de la flota que opera en el Pacífico Oriental se 
efectuó a partir de la información disponible en los registros de la Comisión Interamericana del Atún Tropical CIAT, sobre la flota atunera que opera en el Pacífico Oriental. Sobre ella se precisó los siguientes aspectos:

- Banderas de origen.

- Año de construcción de las embarcaciones más antiguas.

- Año de construcción de las embarcaciones más jóvenes.

- Clasificación de las embarcaciones por año de construcción realizando una sub división en intervalos de 10 años, para fines de cálculos de edades medias por bandera.

Para obtener información detallada sobre la documentación que lleva cada embarcación relacionada a las actividades de mantenimiento y dado la gran cantidad de estas y la imposibilidad de incluir el análisis documental de todas ellas se seleccionó una muestra. Del universo compuesto por 153 embarcaciones que realizan sus operaciones logísticas y de mantenimiento en puertos marítimos ubicados en los cantones Manta y Jaramijó de la Provincia de Manabí
Ecuador, se seleccionó una muestra de 41 embarcaciones lo que equivale a un $27 \%$ del universo. Los criterios para la selección de la muestra fueron la bandera o nacionalidad, eslora total, toneladas de registro bruto y el año de construcción.

De las embarcaciones seleccionadas como muestra de estudio 27 pertenecen a Ecuador, 1 a Colombia,3 a El Salvador, 2 a España, 2 a Estados Unidos, 1 a Nicaragua, 6 a Panamá y 1 a Venezuela que son operadas por 28 empresas armadoras, las dimensiones físicas van de 31,45 hasta $91,90 \mathrm{~m}$ de eslora total y su volumen está enmarcado entre 236 y 3. 005 ton de registro bruto.

Seleccionada la muestra y obtenido el acceso a las bitácoras o diarios de máquinas se procedió a la realización del análisis documental de manera individual y por motores de las variables que son parte de los siguientes sistemas: a) sistema de enfriamiento,

b) sistema de lubricación, c) sistema de admisión de aire, d) sistema de gases de escape, e) sistema de combustible.

Por último con la información de las variables que registran en las 
bitácoras o diarios de máquinas se realizó un análisis de estas coincidencias de las distribuciones de los parámetros registrados entre las embarcaciones muestreadas establecido por porcentajes de similitud.

\section{Resultados y discusión}

Caracterización general de la flota que opera en el Pacífico Oriental

En tabla 1, se detalla la población de buques activos y el año de construcción. En general la flota atunera consta de 280 embarcaciones activas, las cuales se distribuyen por banderas de la siguiente forma: España, Estados Unidos, México, Nicaragua, Panamá, El Salvador, Venezuela, Colombia, Ecuador y Perú. Ecuador posee la mayor cantidad de buques activos, 107 y El Salvador solo 3 embarcaciones. Los buques atuneros industriales más antiguos son de bandera ecuatoriana y se construyeron en el año 1942 y 1946; el tercer buque de mayor antigüedad pertenece a la bandera de Estados Unidos el cual fue construido en el año 1947. Las embarcaciones de edad media pertenecen a las banderas de Nicaragua construidas en el año 1981 y El Salvador con buques construidos en el año 1992; finalmente los buques atuneros industriales con menor años de construcción pertenecen a las banderas de Panamá (2017) y Estados Unidos con una embarcación construida en el año 2018.

Tabla 1. Distribución de embarcaciones del Pacífico Oriental por banderas y años de construcción.

\begin{tabular}{cccc}
\hline País & $\begin{array}{c}\text { Población de } \\
\text { buques activos }\end{array}$ & $\begin{array}{c}\text { Año de construcción } \\
\text { buques de mayor edad }\end{array}$ & $\begin{array}{c}\text { Año de construcción } \\
\text { buques de menor } \\
\text { edad }\end{array}$ \\
\hline Colombia & 14 & 1973 & 2007 \\
\hline Ecuador & 107 & 1942 & 2014 \\
\hline El Salvador & 3 & 1992 & 2015 \\
\hline España & 4 & 1984 & 2003 \\
\hline Estados Unidos & 35 & 1947 & 2018 \\
\hline México & 53 & 1966 & 2017 \\
\hline Nicaragua & 6 & 1971 & 2017 \\
\hline Panamá & 22 & 1970 & 2008 \\
\hline Perú & 16 & 1969 & 2014 \\
\hline Venezuela & 20 & 1971 & \\
\hline Total & $\mathbf{2 8 0}$ & &
\end{tabular}

Fuente: Datos de la Comisión Interamericana del Atún Tropical CIAT (2021). 
En la figura 1 se representa la distribución de embarcaciones por banderas establecidas en porcentajes. Se observa que Ecuador posee el mayor número de embarcaciones activas, que representan un $38 \%$ de la flota que opera en el pacífico oriental, seguido de México con 53 embarcaciones y Estados Unidos con 35 buques activos que representan el $19 \%$ y el $13 \%$ respectivamente. El resto de las banderas tienen menos del $10 \%$ de participación en la flota del pacífico.

Figura 1. Distribución de buques atuneros industriales por bandera.

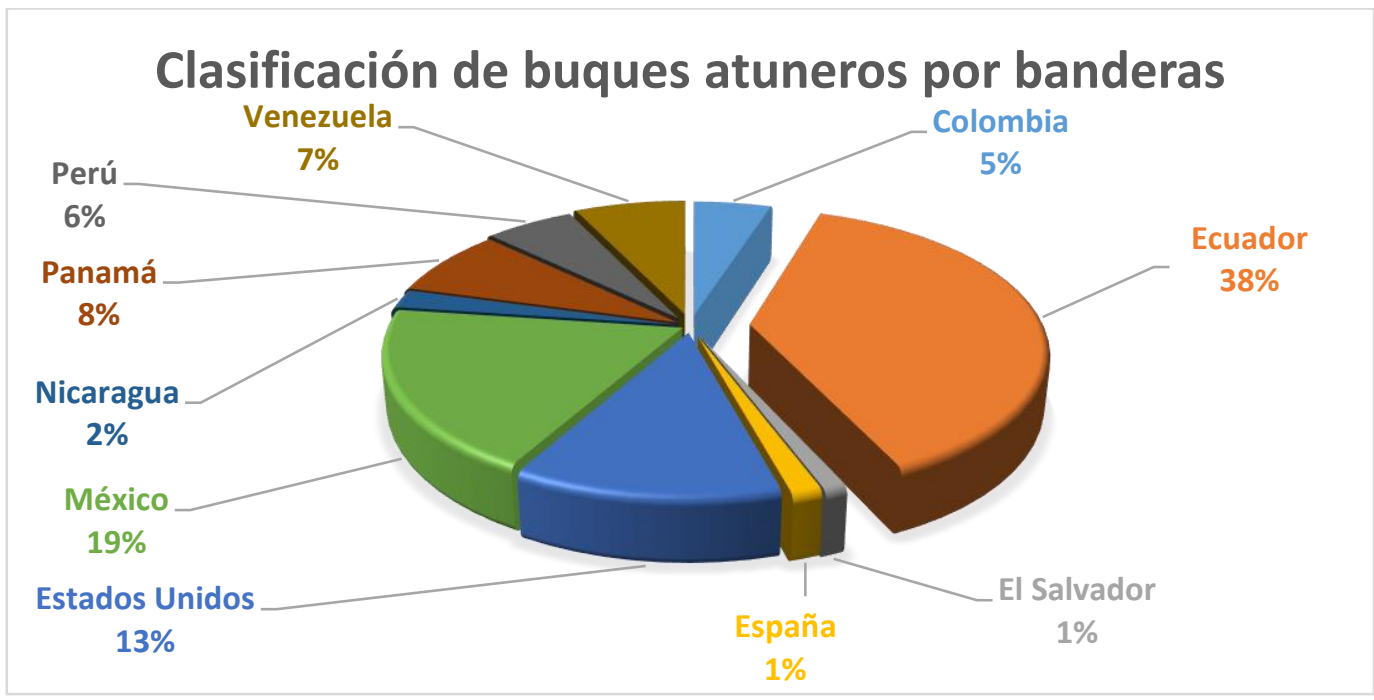

Fuente: Datos de la Comisión Interamericana del Atún Tropical CIAT (2021).

En la figura 2 se representa la distribución de edades para un intervalo de clase de 10 años de las 280 embarcaciones. De ella concluimos que la edad media de la flota atunera que opera en el Pacífico Oriental es de 38 años. Por países la flota salvadoreña es la menos antigua con una edad media de 19 años, seguido de la flota española que presenta una edad media de 25 años. Las flotas más antiguas, que superan los cuarenta años de edad media, son las de Venezuela con 42 años, la de Ecuador con 43 años y por último la de Nicaragua con 46 años. Los resultados mostrados en la tabla 2, se presentan de manera gráfica en la figura 2, la misma que hace énfasis en las flotas que superan los 40 años de edad media. 
Figura 2. Edad media de la flota atunera que opera en el Pacífico Oriental.

\section{Edad media de la flota atunera que opera en el Pacífico Oriental}

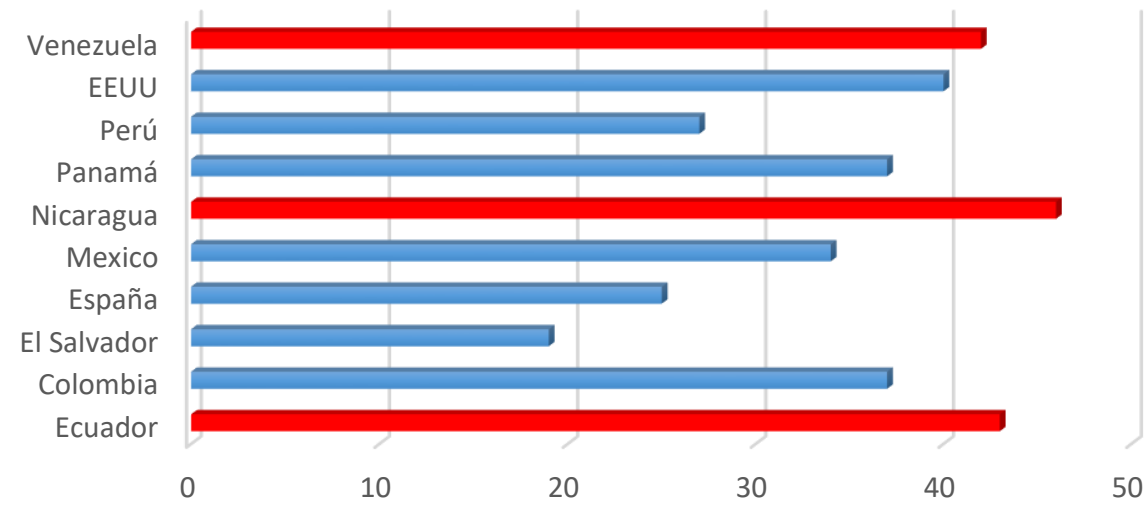

Fuente: Datos de la Comisión Interamericana del Atún Tropical CIAT (2021).

Análisis de las principales variables que registran los buques como parte de la gestión de mantenimiento

En la tabla 2, se muestran para cada una de las variables estudiadas como son sistema de enfriamiento, sistema de lubricación, sistema de admisión de aire, sistema de gases de escape y sistema de combustible, los indicadores que registran las embarcaciones sobre ellas, por ejemplo, en el sistema de enfriamiento registran 19 indicadores lo que lo convierte en el sistema con mayor cantidad de datos registrados, seguido del sistema de lubricación con 9 indicadores, sistema de admisión de aire con 3 indicadores y por último encontramos al sistema de gases de escape y al sistema de combustible con 3 indicadores cada uno.

Tabla 2. Distribución de indicadores registrados de los sistemas de los motores

\begin{tabular}{ccccc}
\hline Sistema de Enfriamiento & $\begin{array}{c}\text { Sistema de } \\
\text { Lubricación }\end{array}$ & $\begin{array}{c}\text { Sistema de } \\
\text { Admisión de } \\
\text { Aire }\end{array}$ & $\begin{array}{c}\text { Sistema de } \\
\text { Gases de } \\
\text { Escape }\end{array}$ & $\begin{array}{c}\text { Sistema de } \\
\text { Combustible }\end{array}$ \\
\hline $\begin{array}{c}\text { Temperatura de Agua de } \\
\text { Entrada Enfriador de Aceite }\end{array}$ & $\begin{array}{c}\text { Presión de } \\
\text { Aceite de } \\
\text { Retorno }\end{array}$ & $\begin{array}{c}\text { Presión de } \\
\text { Aire de } \\
\text { Turbos }\end{array}$ & $\begin{array}{c}\text { Temperatura } \\
\text { de Gases de } \\
\text { Escape }\end{array}$ & $\begin{array}{c}\text { Consumo de } \\
\text { Combustible }\end{array}$ \\
\hline $\begin{array}{c}\text { Temperatura de Agua } \\
\text { Salida Enfriador de Aceite }\end{array}$ & $\begin{array}{c}\text { Presión de } \\
\text { Aceite }\end{array}$ & $\begin{array}{c}\text { Temperaura } \\
\text { de Turbos }\end{array}$ & $\begin{array}{c}\text { Presión de } \\
\text { Gases de } \\
\text { Salida del } \\
\text { Turbo }\end{array}$ & $\begin{array}{c}\text { Presión de } \\
\text { Combustible }\end{array}$ \\
\hline
\end{tabular}




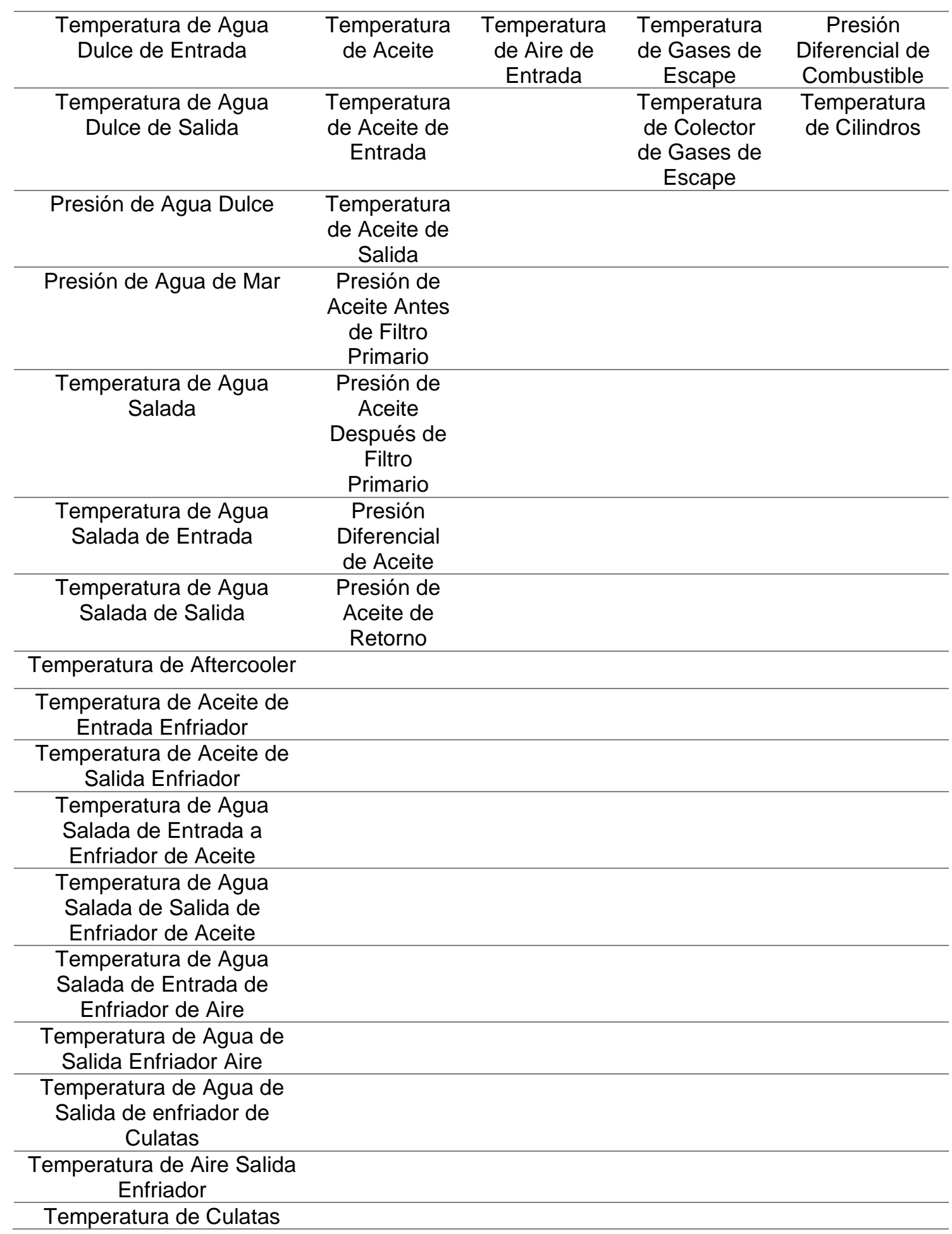

Fuente: Buques atuneros investigados.

En la tabla 3 se reflejan los mayor frecuencia de monitoreo es la resultados obtenidos en el análisis temperatura de agua dulce de del sistema de enfriamiento, entrada al motor representado por el concluyendo que el indicador con $85,40 \%$ de la muestra, seguido de la 
presión de agua de mar representado por el $68,30 \%$ y por la temperatura de agua dulce de salida de motor, presión de agua dulce y temperatura de agua de mar, que son medidos por el $48,80 \%$ de las embarcaciones seleccionadas para muestra de estudio, por lo que se consideran los más significativos.

Tabla 3. Análisis de coincidencia de indicadores registrados entre embarcaciones del sistema de enfriamiento

\begin{tabular}{lcc}
\hline \multicolumn{1}{c}{$\begin{array}{c}\text { Parámetros Principales del Sistema de } \\
\text { Enfriamiento }\end{array}$} & $\begin{array}{c}\mathbf{N}^{\circ} \\
\text { Embarcaciones }\end{array}$ & $\begin{array}{c}\% \text { de } \\
\text { coincidencia }\end{array}$ \\
\hline $\begin{array}{l}\text { Temperatura de Agua Dulce de Entrada Enfriador de } \\
\text { Aceite }\end{array}$ & 4 & $9,80 \%$ \\
\hline $\begin{array}{l}\text { Temperatura de Agua Dulce de Salida Enfriador de } \\
\text { Aceite }\end{array}$ & $4,80 \%$ \\
\hline Temperatura de Agua Dulce de Entrada a Motor & 35 & $85,40 \%$ \\
\hline Temperatura de Agua Dulce de Salida de Motor & 20 & $48,80 \%$ \\
\hline Presión de Agua Dulce & 20 & $48,80 \%$ \\
\hline Presión de Agua de Mar & 28 & $68,30 \%$ \\
\hline Temperatura de Agua de Mar & 20 & $48,80 \%$ \\
\hline Temperatura de Agua de Mar de Entrada & 8 & $19,50 \%$ \\
\hline Temperatura de Agua de Mar de Salida & 4 & $9,80 \%$ \\
\hline Temperatura de Aftercooler & 4 & $9,80 \%$ \\
\hline Temperatura de Aceite de Entrada Enfriador & 8 & $19,50 \%$ \\
\hline Temperatura de Aceite de Salida Enfriador & 8 & $19,50 \%$ \\
\hline $\begin{array}{l}\text { Temperatura de Agua de Mar de Entrada a Enfriador } \\
\text { de Aceite }\end{array}$ & 4 & $9,80 \%$ \\
\hline $\begin{array}{l}\text { Temperatura de Agua de Mar de Salida de Enfriador } \\
\text { de Aceite }\end{array}$ & 4 & $9,80 \%$ \\
\hline $\begin{array}{l}\text { Temperatura de Agua de Mar de Entrada de Enfriador } \\
\text { de Aire }\end{array}$ & 8 & \\
\hline Temperatura de Agua de Mar de Salida Enfriador Aire & 8 & $19,50 \%$ \\
\hline Temperatura de Agua de Mar de enfriador de Culatas & 8 & $9,80 \%$ \\
\hline Temperatura de Aire Salida Enfriador & 4 & $19,50 \%$ \\
\hline Temperatura de Culatas & 4 & $19,50 \%$ \\
\hline
\end{tabular}

Fuente: Bitácoras de buques atuneros investigados

En la figura 3 se representan en forma gráfica los parámetros monitoreados del sistema de enfriamiento con mayor frecuencia entre embarcaciones. 
Figura 3. Gráfica de frecuencia de indicadores registrados del sistema de enfriamiento

\section{SISTEMA DE ENFRIAMIENTO}

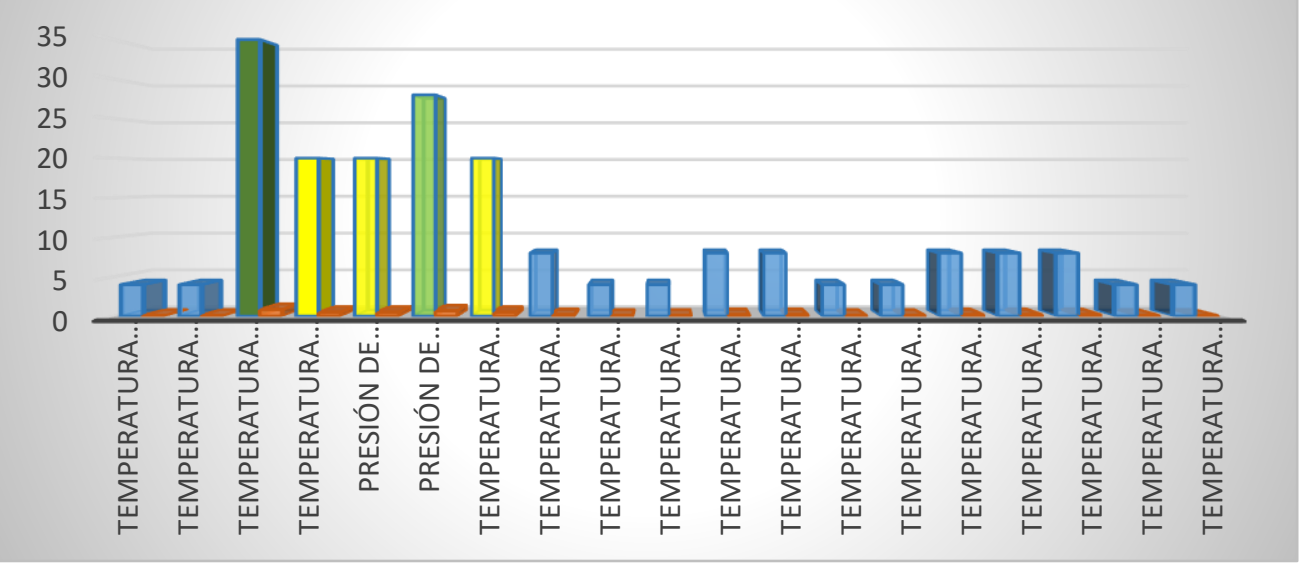

Fuente: Bitácoras de buques atuneros investigados.

Con respecto a los resultados obtenidos en el análisis del sistema de lubricación, en la tabla 4 se detalla que la presión de aceite es relevante en el $100 \%$ de las embarcaciones seleccionadas para muestra de estudio, seguido de la temperatura de aceite que es registrado por el $78 \%$ de las embarcaciones, también evidenciamos que el $59 \%$ de las embarcaciones registran como variable a la temperatura de aceite de salida.

Tabla 4. Análisis de coincidencia de indicadores registrados entre embarcaciones del sistema de lubricación.

\begin{tabular}{lcc}
\hline \multicolumn{1}{c}{$\begin{array}{c}\text { Parámetros principales del Sistema de } \\
\text { Lubricación }\end{array}$} & $\begin{array}{c}\mathbf{N}^{\circ} \text { de } \\
\text { embarcaciones }\end{array}$ & $\begin{array}{c}\text { \% de } \\
\text { coincidencia }\end{array}$ \\
\hline Presión de Aceite de Retorno & 4 & $10 \%$ \\
\hline Presión de Aceite & 41 & $100 \%$ \\
\hline Temperatura de Aceite & 12 & $29 \%$ \\
\hline Temperatura de Aceite de Entrada & 32 & $78 \%$ \\
\hline Temperatura de Aceite de Salida & 24 & $59 \%$ \\
\hline Presión de Aceite Antes de Filtro Primario & 8 & $20 \%$ \\
\hline Presión de Aceite Después de Filtro Primario & 8 & $20 \%$ \\
\hline Presión Diferencial de Aceite & 4 & $10 \%$ \\
\hline
\end{tabular}

Elaboración: Ing. José Adrián Zambrano Ascencio (Autor de la investigación).

Fuente: Bitácoras de buques atuneros investigados.

En la figura 4, se representan de forma gráfica los indicadores monitoreados del sistema de lubricación con mayor frecuencia entre las embarcaciones. 
Figura 4. Gráfica de frecuencia de indicadores registrados del sistema de lubricación.

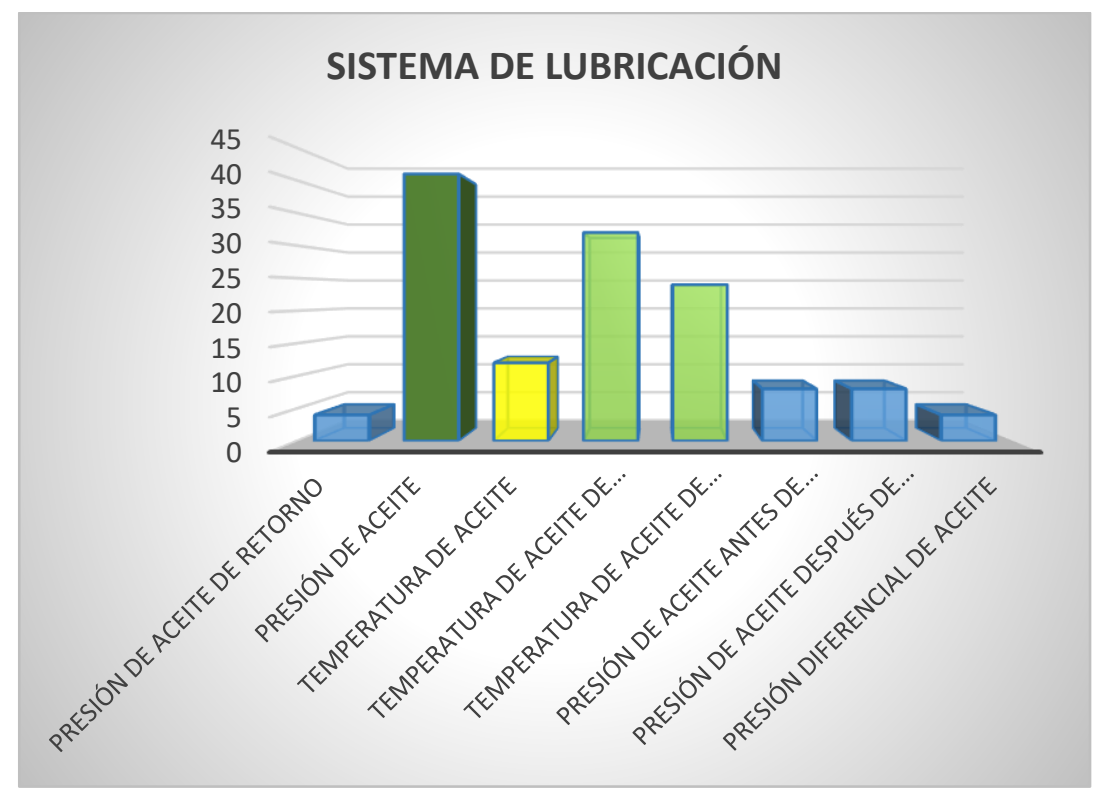

Fuente: Bitácoras de buques atuneros investigados.

En la tabla 5 se detallan los temperatura de aire de entrada resultados obtenidos en el análisis tienen una frecuencia de registro del sistema de admisión de aire, menor al $40 \%$ entre las concluyendo que la presión de aire embarcaciones seleccionadas para de turbos, temperatura de turbos y muestra de estudio.

Tabla 5. Análisis de coincidencia de indicadores registrados entre embarcaciones del sistema de admisión de aire.

\begin{tabular}{lcc}
\hline $\begin{array}{c}\text { Parámetros Principales del Sistema de } \\
\text { Admisión de Aire }\end{array}$ & $\begin{array}{c}\mathbf{N}^{\circ} \text { de } \\
\text { embarcaciones }\end{array}$ & $\begin{array}{c}\% \text { de } \\
\text { coincidencia }\end{array}$ \\
\hline Presión de Aire de Turbos & 12 & $29 \%$ \\
\hline Temperatura de Turbos & 16 & $39 \%$ \\
\hline Temperatura de Aire de Entrada & 16 & $39 \%$ \\
\hline
\end{tabular}

Elaboración: Ing. José Adrián Zambrano Ascencio (Autor de la investigación).

Fuente: Bitácoras de buques atuneros investigados.

En la figura 5, se representan de forma gráfica los parámetros monitoreados del sistema de admisión de aire. 
Figura 5. Gráfica de frecuencia de indicadores registrados del sistema de admisión de aire.

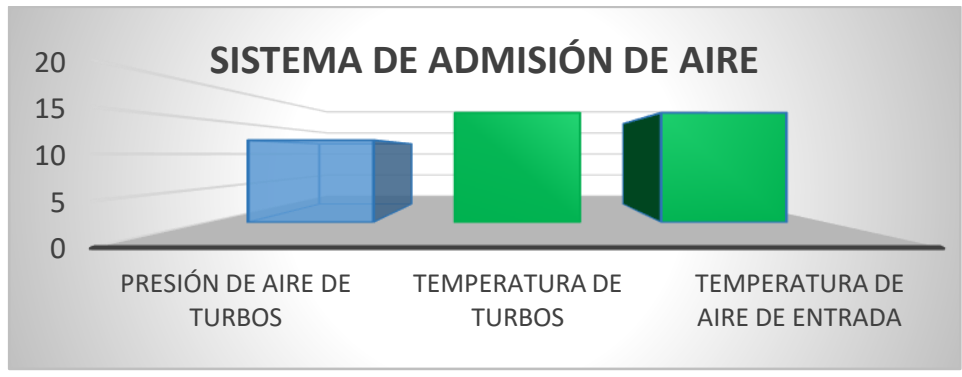

Fuente: Bitácoras de buques atuneros investigados.

En la tabla 6 se evidencian los resultados obtenidos en el análisis del sistema de gases de escape, concluyendo que la temperatura de gases de escape, presión de gases de salida del turbo y la temperatura de colector de gases de escape presentan una frecuencia de registro no mayor al $12 \%$ entre las embarcaciones seleccionadas para muestra de estudio.

Tabla 6. Análisis de coincidencia de indicadores registrados entre embarcaciones del sistema de gases de escape

\begin{tabular}{lcc}
\hline $\begin{array}{c}\text { Parámetros principales del Sistema } \\
\text { de Gases de Escape }\end{array}$ & $\begin{array}{c}\mathbf{N}^{\circ} \text { de } \\
\text { Embarcaciones }\end{array}$ & $\begin{array}{c}\% \text { de } \\
\text { coincidencia }\end{array}$ \\
\hline Temperatura de Gases de Escape & 5 & $12 \%$ \\
\hline Presión de Gases de Salida del Turbo & 3 & $7 \%$ \\
\hline $\begin{array}{l}\text { Temperatura de Colector de Gases de } \\
\text { Escape }\end{array}$ & 1 & $2 \%$ \\
\hline
\end{tabular}

Elaboración: Ing. José Adrián Zambrano Ascencio (Autor de la investigación).

Fuente: Bitácoras de buques atuneros investigados.

En la figura 6, se representan las cantidades de embarcaciones que coinciden en la captura de parámetros del sistema de admisión de gases de escape.

Figura 6. Gráfica de frecuencia de indicadores registrados del sistema de gases de escape.

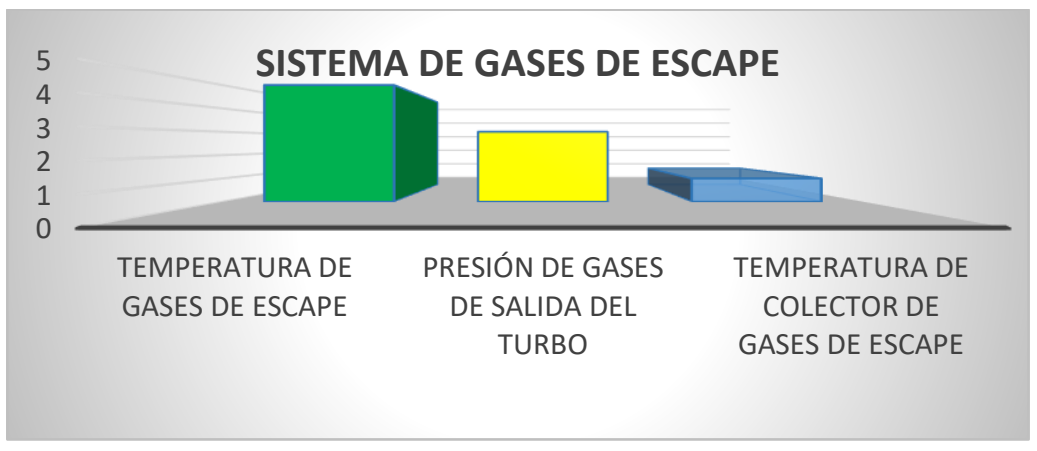

Fuente: Bitácoras de buques atuneros investigados. 
En la tabla 7, se reflejan los resultados obtenidos en el análisis del sistema de combustible, que a diferencia de los sistemas de admisión de aire y de gases de escape, posee indicadores como presión y consumo de combustible, los mismos que se consideran relevantes debido a que presentan un indicador de frecuencia equivalente al $100 \%$ de la muestra de estudio.

Tabla 7. Análisis de coincidencia de indicadores registrados entre embarcaciones del sistema de combustible.

\begin{tabular}{lcc}
\hline $\begin{array}{c}\text { Parámetros principales del Sistema de } \\
\text { Combustible }\end{array}$ & $\begin{array}{c}\mathbf{N}^{\circ} \text { de } \\
\text { Embarcaciones }\end{array}$ & $\begin{array}{c}\% \text { de } \\
\text { coincidencia }\end{array}$ \\
\hline Consumo de Combustible & 41 & $100 \%$ \\
\hline Presión de Combustible & 41 & $100 \%$ \\
\hline Presión Diferencial de Combustible & 4 & $10 \%$ \\
\hline Temperatura de Cilindros & 8 & $20 \%$ \\
\hline
\end{tabular}

Elaboración: Ing. José Adrián Zambrano Ascencio (Autor de la investigación).

Fuente: Bitácoras de buques atuneros investigados.

En la figura 7 , se representan las parámetros del sistema de cantidades de embarcaciones que combustible.

coinciden en la captura de

Figura 7. Gráfica de frecuencia de indicadores registrados del sistema de combustible.

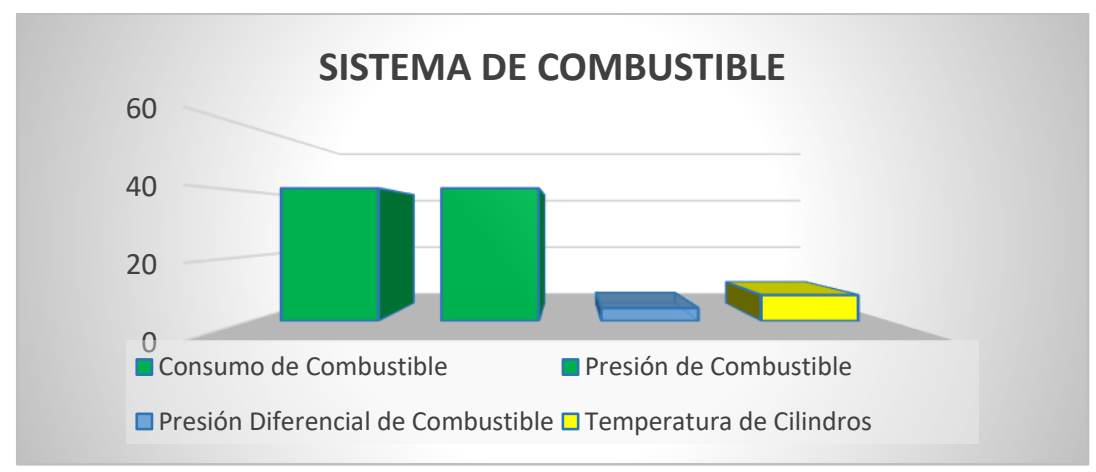

Fuente: Bitácoras de buques atuneros investigados.

\section{Conclusiones}

En función de la caracterización de la flota atunera industrial que opera en el pacífico oriental se concluye que está conformada por 280 embarcaciones activas distribuidas en 10 países y su edad media es de 38 años, siendo la flota ecuatoriana la más numerosa con 107 buques activos con una edad media de 43 años tomando como base que el 80 $\%$ de sus embarcaciones superan 
los 40 años de antigüedad y que posee dos embarcaciones con edades superiores a 70 años. La flota de El Salvador resulta ser la menos numerosa con solo 3 embarcaciones activas y es considerada como la más joven por su edad media equivalente a 19 años. Adicional concluimos que la flota nicaragüense y venezolana son las más antiguas debido a que sus edades medias son de 46 y 42 años respectivamente.

En función del análisis documental realizado a las embarcaciones seleccionadas para muestra de estudio concluimos que el sistema o variable que está sujeta a un mayor control se direcciona al sistema de enfriamiento debido a que se registran 19 indicadores, de los cuales, la temperatura da agua dulce de entrada al motor fue el indicador más utilizado de dicho sistema en las embarcaciones, sin embargo, ninguno de los indicadores del sistema de enfriamiento estuvo presente en todas las embarcaciones que conformaron la muestra de estudio. Indicadores como presión de aceite, presión de combustible y consumo de combustible estuvieron presentes en el $100 \%$ de la muestra de estudio, lo que indica que son considerados los más útiles como herramienta de mantenimiento predictivo, mientras que en los sistemas de admisión de aire y gases de escape se registran muy pocos indicadores y con un factor de coincidencia muy bajo entre embarcaciones.

Como resultado general de la presente investigación se logró la caracterización de la flota atunera que opera en el Pacífico Oriental y en esencia concluimos lo siguiente: Se trata de una flota antigua incluyendo sus motores diésel y resto de equipos instalados a bordo; no dispone de tecnologías actualizadas de monitoreo por lo que los datos son registrados manualmente en bitácoras físicas; el monitoreo se fundamenta en el registro de indicadores y los más relevantes resultaron ser la presión de aceite y la presión y consumo de combustible, el resto de indicadores se utilizan con mediana o poca frecuencia como herramienta de control. 


\section{Bibliografía}

Castillo, S. A., y Serrano, J. R. (2016). metodología De Mantenimiento Predictivo Para Diagnostico De Fallas En Bombas Hidráulicas De Pistones Axiales Asociadas Con Desgastes De Los Platos Rodantes De etodol. Universidad Industrial de Santander, Escuela De Ingetodolo.

Cheliotis., M. Lazakis., I. y Theotokatos., G. (2020). Machine learning and datadriven fault detection for ship systems operations, Ocean Engineering, Volume 216.

Delgado, A. Guevara, C. (2014) p. 81 Análisis de los procedimientos de mantenimiento en el buque Tohalli del Instituto Nacional de Pesca de la ciudad de Guayaquil y su influencia en el tiempo de vida útil de los equipos.

Dikis, K., Lazakis, I., \& Theotokatos, G. (2015). Dynamic reliability analysis tool for ship machinery maintenance.

EXTERIOR, M. D. C. (2017). REPORTE DEL SECTOR ATUNERO ECUATORIANO.

FAO. (2018). La Agricultura y el estado mundial de la Pesca.

García Rodríguez, J. (2016). Proyecto de mejora en el plan de mantenimiento de la barcaza aljibe" Santa Cruz".
González R, (2012) Diseño de un plan de Mantenimiento para una embarcación de 32 metros, para optar al título de grado en Ingeniera Marina, Escuela Técnica Superior de Náutica, Universidad de Cantabria, Cantabria, 2012, p.53-P. 27-28.

Hernández, E. (2021) Ingeniería CBM: Monitoreo basado en condición estándar ISO 17359

Jaramillo, V., Noureddine, B. y Haugen., H. (2020). Developing a predictive maintenance model for vessel machinery. Journal of Ocean Engineering and Science. Volume 5, Issue 4, December 2020, Pages 358-386.

Jones NB, Li Yu-Hua. A review of condition monitoring and fault diagnosis for diesel engines. Tribotest Int J 2000;6(3):26791.

Moreno Cayuela, S. (2016). Implantación de un sistema de mantenimiento predictivo basado en condición en una plataforma nava.

Pacheco, J. (2019). Proceso de Investigación de los Recursos Bioacuáticos y su Ambiente.

Martínez, I. D y Vanegas, J. A. (2017). definición de la estrategia de mantenimiento basado en condición (CBM) soportada en técnicas de mantenimiento predictivo, para los equipos críticos de la operación de campo 
Velásquez ubicado en el municipio de Puerto Boyacá.

Universidad Industrial de

Santander, Escuela De Ing.

Mecánica.

Pesantes Carlos, (2011) Análisis y

Mejora del Plan de

Mantenimiento del Buque con

/RO OPDR ANDALICIA,

Santa Cruz de Tenerife, p.47.

Raptodimos, Y., Lazakis, I., Theotokatos, G., Salinas, R., \& Moreno, A. (2016). Collection and analysis of data for ship condition monitoring aiming at enhanced reliability and safety.

Solís, J. A. (2018). Antigüedad y Mantenimiento: Desafío para la Flota Atunera Ecuatoriana. 